\title{
ASYMPTOTICALLY POWER SOLUTIONS OF HIGHER ORDER NEUTRAL FUNCTIONAL DIFFERENTIAL EQUATIONS
}

\author{
ZHI-QIANG ZHU AND SUI SUN CHENG
}

\begin{abstract}
Necessary and sufficient conditions are derived for the existence of asymptotically polynomial solutions of a class of neutral functional differential equations.
\end{abstract}

In $[1,2], n$-th order neutral functional differential equations of the form

$$
(x(t)+c(t) x(t-d))^{(n)}+f\left(t, x\left(g_{1}(t)\right), x\left(g_{2}(t)\right), \cdots, x\left(g_{m}(t)\right)\right)=0, t \geq t_{0},
$$

are investigated and necessary and sufficient conditions for the existence of nonoscillatory solutions are derived. We observed that in these papers, the condition that $c(t) \geq-1$ is assumed. The question then arises as to whether existence criteria can be established in case $c(t)<-1$.

In this note, we will show that under the basic assumptions that $f$ is either superlinear or sublinear and that $\lim _{t \rightarrow \infty} c(t)=c_{0}<-1$, necessary and sufficient conditions can be found for the existence of "asymptotically power" solutions.

More precisely, let $d>0, h>0, m \in\{1,2, \ldots\}$ and $n \in\{2,3, \ldots\}$. Let $c \in$ $C\left(\left[t_{0},+\infty\right), R\right)$ and $\lim _{t \rightarrow+\infty} c(t)=c_{0}$. We assume that $g_{1}, g_{2}, \ldots, g_{m} \in C\left(\left[t_{0},+\infty\right), R\right)$ and satisfy $g_{1}(t), \ldots, g_{m}(t) \geq t-h$ for some constant $h>0, f\left(t, x_{1}, x_{2}, \ldots, x_{m}\right) \in$ $C\left(\left[t_{0},+\infty\right) \times R^{m}, R\right)$, and,

$$
x_{1} f\left(t, x_{1}, x_{2}, \ldots, x_{m}\right)>0, t \geq t_{0},
$$

if $x_{1} x_{i}>0$ for $i=1,2, \ldots, m$.

A function $f\left(t, x_{1}, x_{2}, \ldots, x_{m}\right)$ is said to be superlinear if there exist continuous functions $p_{i}(t) \geq 0$ for $i=1,2, \ldots, m$, satisfying $\sum_{i=0}^{m} p_{i}(t)>0$ and

$$
\frac{f\left(t, y_{1}, y_{2}, \ldots, y_{m}\right)}{\sum_{i=0}^{m} p_{i}(t) y_{i}} \geq \frac{f\left(t, x_{1}, x_{2}, \ldots, x_{m}\right)}{\sum_{i=0}^{m} p_{i}(t) x_{i}}
$$

when $y_{i} \geq x_{i}>0$ or $y_{i} \leq x_{i}<0$ for $i=1,2, \ldots, m$. If the inequality $\geq$ in (2) is changed into $\leq$, then the function $f\left(t, x_{1}, x_{2}, \ldots, x_{m}\right)$ is said to be sublinear.

Received October 29, 2003.

2000 Mathematics Subject Classification. 34C10.

Key words and phrases. Power function solution, neutral functional differential equation, Krasnoselskii theorem. 
The following is shown in [1] which can also be shown easily.

Lemma 1. Suppose that $0<a \leq x_{i} \leq b$ for $i=1,2, \ldots, m$. If $f$ is superlinear, then

$$
f(t, a, \ldots, a) \leq f\left(t, x_{1}, \ldots, x_{m}\right) \leq f(t, b, \ldots, b), t \geq t_{0} .
$$

If $f$ is sublinear, then

$$
\frac{a}{b} f(t, b, \ldots, b) \leq f\left(t, x_{1}, \ldots, x_{m}\right) \leq \frac{b}{a} f(t, a, \ldots, a), t \geq t_{0} .
$$

Lemma 2. Suppose that $\lim _{t \rightarrow+\infty} c(t)=c_{0} \neq \pm 1$ and that $x(t) / t^{i}$ is bounded and eventually positive or eventually negative, where $i$ is a nonnegative integer. Let $z(t)=$ $x(t)+c(t) x(t-d)$. If $\lim _{t \rightarrow+\infty} z(t) / t^{i}=b$ exists, then $\lim _{t \rightarrow+\infty} x(t) / t^{i}=b /\left(1+c_{0}\right)$.

The proof is similar to that of Lemma 2 in [1] and is ommited.

Lemma 3.(Krasnoselskii [1]) Suppose $B$ is a Banach space and $X$ is a bounded, convex and closed subset of $B$. Let $U, S: X \rightarrow B$ satisfy the following conditions:

(i) $U x+$ Sy for any $x, y \in X$,

(ii) $U$ is a contraction mapping, and

(iii) $S$ is completely continuous.

Then $U+S$ has a fixed point in $X$.

As usual, a solution $x(t)$ of $(1)$ is said to be nonoscillatory if it eventually positive or eventually negative. A nonoscillatory solution $x(t)$ of (1) is said to be asymptotically constant or belong to $T_{0}(a)$ if

$$
\lim _{t \rightarrow+\infty} x(t)=a \neq 0 .
$$

Two natural extensions of the concept of an asymptotically constant solution can be stated as follows. A nonoscillatory solution $x(t)$ of $(1)$ is said to belong to $T_{r}(\infty, a)$, where $r \in\{1,2, \ldots, n-1\}$, if

$$
\limsup _{t \rightarrow+\infty} \frac{|x(t)|}{t^{r-1}}=+\infty \text { and } \limsup _{t \rightarrow+\infty} \frac{|x(t)|}{t^{r}}=a \neq 0
$$

and said to belong to $T_{r}(a, 0)$, where $r \in\{1,2, \ldots, n-1\}$, if

$$
\limsup _{t \rightarrow+\infty} \frac{|x(t)|}{t^{r-1}}=a \neq 0 \text { and } \limsup _{t \rightarrow+\infty} \frac{|x(t)|}{t^{r}}=0 .
$$

We will be interested in finding necessary and sufficient conditions for the existence of solutions in $T_{0}(a), T_{r}(\infty, a)$ and $T_{r}(a, 0)$.

Theorem 1. Suppose $\lim _{t \rightarrow+\infty} c(t)=c_{0}<-1$ and $f$ is either superlinear or sublinear. Then (1) has a nonoscillatory solution $x(t) \in T_{r}(\infty, a)$ if, and only if, there is some $K \neq 0$ such that

$$
\int_{t_{0}}^{+\infty} s^{n-r-1}\left|f\left(s, K g_{1}^{r}(s), K g_{2}^{r}(s), \ldots, K g_{m}^{r}(s)\right)\right| d s<+\infty .
$$


If $r=0, T_{r}(\infty, a)$ can be replaced by $T_{0}(a)$ in the above statement.

Proof. Let

$$
G_{x}(t)=f\left(t, x\left(g_{1}(t)\right), x\left(g_{2}(t)\right), \ldots, x\left(g_{m}(t)\right)\right)
$$

and

$$
F_{r}(t)=f\left(t, K g_{1}^{r}(t), K g_{2}^{r}(t), \ldots, K g_{m}^{r}(t)\right)
$$

Since

$$
\int_{\alpha}^{x}(x-y)^{n} g(y) d y=n ! \int_{\alpha}^{x} d y_{n} \int_{\alpha}^{y_{n}} d y_{n-1} \cdots \int_{\alpha}^{y_{2}} d y_{1} \int_{\alpha}^{y_{1}} g(y) d y,
$$

(3) is equivalent to

$$
\int_{t_{0}}^{+\infty} \int_{s_{n-r-1}}^{+\infty} \cdots \int_{s_{1}}^{+\infty}\left|F_{r}(s)\right| d s d s_{1} \cdots d s_{n-r-1}<+\infty .
$$

Let $x(t)$ be an eventually positive solution of $(1)$ in $T_{r}(\infty, a)$. Then without loss of generality, we may suppose there exists $T>t_{0}$ such that $x(t)>0, x(t-d)>0$ and $x\left(g_{i}(t)\right)>0$ for $t \geq T$ and $i=1,2, \ldots, m$. Let

$$
z(t)=x(t)+c(t) x(t-d) .
$$

Then, by (1), we have

$$
z^{(n)}(t)=-f\left(t, x\left(g_{1}(t)\right), x\left(g_{2}(t)\right), \ldots, x\left(g_{m}(t)\right)\right) .
$$

In view of $(1)$, we have $z^{(n)}(t)<0$ for $t \geq T$. Therefore $z^{(i)}(t)$ is eventually monotonic for all $i \in\{0,1,2, \ldots, n-1\}$. Since $\lim _{t \rightarrow+\infty} \frac{x(t)}{t^{r}}=a>0$, there exists $T_{1} \geq T$ such that

$$
\frac{1}{2} a t^{r} \leq x(t) \leq \frac{3}{2} a t^{r}, t \geq T_{1} \text {. }
$$

Noticing $\lim _{t \rightarrow+\infty} \frac{z(t)}{t^{r}}=\left(1+c_{0}\right) a$, we have

$$
\lim _{t \rightarrow+\infty} z^{(r)}(t)=\left(1+c_{0}\right) a r !
$$

Invoking the monotonicity of $z^{(i)}(t)$ and (9), we have

$$
\lim _{t \rightarrow+\infty} z^{(i)}(t)=0, i=r+1, r+2, \ldots, n-1 .
$$

After integrating (7) $n-r-1$ times, we obtain

$$
z^{(r+1)}(t)=(-1)^{n-r} \int_{t}^{+\infty} \int_{s_{n-r-2}}^{+\infty} \cdots \int_{s_{1}}^{+\infty} G_{x}(s) d s d s_{1} \cdots d s_{n-r-2}, t \geq T_{1} .
$$

Then integrating the above formula from $T_{1}$ to $t$, we obtain

$$
z^{(r)}(t)=z^{(r)}(T)+(-1)^{n-r} \int_{T_{1}}^{t} \int_{s_{n-r-1}}^{+\infty} \int_{s_{n-r-2}}^{+\infty} \ldots \int_{s_{1}}^{+\infty} G_{x}(s) d s d s_{1} \ldots d s_{n-r-1} .
$$


In view of (9), we have

$$
\int_{T_{1}}^{+\infty} \int_{s_{n-r-1}}^{+\infty} \int_{s_{n-r-2}}^{+\infty} \ldots \int_{s_{1}}^{+\infty} G_{x}(s) d s d s_{1} \ldots d s_{n-r-1}<+\infty .
$$

In view of Lemma 1 and (8), we see that $F_{r}(t) \leq G_{x}(t)$ where we set $K=a / 2$ if $f$ is superlinear, and $F_{r}(t) \leq 3 G_{x}(t)$ where we set $K=3 a / 2$ if $f$ is sublinear. In view of (11), we see that $(7)$ holds when $x(t)$ is eventually positive.

The case that $x(t)$ is eventually negative can be proved in a similar manner.

Conversely, suppose that $K>0$. Let $e=K / 2$ if $f$ is superlinear and $e=K$ if $f$ is sublinear. Set $R(t)=t^{r}$ or $R(t) \equiv 1$ when $r=0$. Take $c_{1}$ and $c_{2}$ so that $\left(-8 c_{0}-1\right) / 7>c_{2}>\left|c_{0}\right|>c_{1}>1$. Then $c_{0}<-\left(7 c_{2}+1\right) / 8$. Since

$$
\lim _{t \rightarrow+\infty} \frac{7-7 c_{2}-8 c(t+d)}{8}=\frac{7-7 c_{2}-8 c_{0}}{8}>1
$$

and

$$
\lim _{t \rightarrow+\infty}\left(c(t)+c_{2}\right)=c_{0}+c_{2}<\frac{1}{8}\left(c_{2}-1\right),
$$

there exists a sufficiently large $T>t_{0}+h+d$ such that when $t \geq T$, we have

$$
\begin{gathered}
\frac{1}{|c(t+d)|} \frac{R^{2}(t+d)}{R^{2}(t-d-h)} \leq \frac{1}{c_{1}}, \\
|c(t)| \geq c_{1},|c(t)| \leq c_{2}, \\
c(t)+c_{2} \leq \frac{1}{8}\left(c_{2}-1\right), \\
\frac{R(t+d)}{R(t)} \leq \frac{7-7 c_{2}-8 c(t+d)}{8},
\end{gathered}
$$

and

$$
\int_{T}^{+\infty} \int_{s_{n-r-1}}^{+\infty} \int_{s_{n-r-2}}^{+\infty} \ldots \int_{s_{1}}^{+\infty} F_{r}(s) d s d s_{1} \ldots d s_{n-r-1}<\frac{c_{2}-1}{8} e .
$$

Take $\bar{T}=T-d-h$ and the linear space

$$
C_{R}[\bar{T},+\infty)=\left\{x \in C([\bar{T},+\infty), R): \sup _{t \geq \bar{T}} \frac{|x(t)|}{R^{2}(t)}<+\infty\right\}
$$

with norm $\|x\|_{R}=\sup _{t \geq \bar{T}} \frac{|x(t)|}{R^{2}(t)}$. Then $C_{R}[\bar{T},+\infty)$ is a Banach space. Set

$$
X=\{x \in[\bar{T},+\infty): e R(t) \leq x(t) \leq 2 e R(t)\}
$$

Then it is obvious that $X$ is a bounded convex and closed subset of $C_{R}[\bar{T},+\infty)$ and for any $x \in X$ and $t \geq \bar{T}+h$,

$$
G_{x}(t) \leq 2 F_{r}(t)
$$


Define two operators on $X$ as follows:

$$
(U x)(t)= \begin{cases}\frac{3 e R(t)}{2 c(T+d)}-\frac{1}{c(T+d)} \frac{x(T+d)}{R(T)} R(t) & \bar{T} \leq t<T \\ \frac{3 e R(t)}{2 c(t+d)}-\frac{1}{c(t+d)} x(t+d) & t \geq T\end{cases}
$$

and

$$
(S x)(t)= \begin{cases}-\frac{3 c_{2} e R(t)}{2 c(T+d)} & \bar{T} \leq t<T \\ -\frac{3 c_{2} e R(t)}{2 c(t+d)}+\frac{(-1)^{n-r-1}}{c(t+d)} H(t) & t \geq T\end{cases}
$$

where

$$
H(t)=\int_{T+d}^{t+d} \int_{T}^{s_{n-1}} \cdots \int_{T}^{s_{n-r+1}} \int_{s_{n-r}}^{+\infty} \ldots \int_{s_{1}}^{+\infty} G_{x}(s) d s d s_{1} \ldots d s_{n-1} .
$$

We will show that the operator $U$ and $S$ satisfy the conditions of the Krasnoselskii fixed point theorem.

(i) First we assert that $U x+S y \in X$ for any $x, y \in X$. Indeed, for $t \in[\bar{T}, T)$, in view of (13) and (15), we have

$$
\begin{aligned}
(U x)(t)+(S y)(t) & =\left(\frac{3\left(1-c_{2}\right)}{2 c(T+d)} e R(T)-\frac{x(T+d)}{c(T+d)}\right) \frac{R(t)}{R(T)} \\
& \geq\left(\frac{3\left(1-c_{2}\right)}{2 c(T+d)} e R(T)-\frac{e R(T+d)}{c(T+d)}\right) \frac{R(t)}{R(T)} \\
& \geq e R(t),
\end{aligned}
$$

and

$$
\begin{aligned}
(U x)(t)+(S y)(t) & \leq\left(\frac{3\left(1-c_{2}\right)}{2 c(T+d)} e R(T)-\frac{2 e R(T+d)}{c(T+d)}\right) \frac{R(t)}{R(T)} \\
& =\left(\frac{3\left(1-c_{2}\right)}{2 c(T+d)}-\frac{2}{c(T+d)} \frac{R(T+d)}{R(T)}\right) e R(t) \\
& \leq\left(\frac{3\left(1-c_{2}\right)}{2 c(T+d)}-\frac{1}{c(T+d)} \frac{7-7 c_{2}-8 c(T+d)}{4}\right) \frac{R(t)}{R(T)} \\
& \leq 2 e R(t) .
\end{aligned}
$$

When $t \in[T,+\infty)$, in view of (16) and (17), we have

$$
\int_{T}^{t} \int_{T}^{s_{n-1}} \ldots \int_{T}^{s_{n-r+1}} \int_{s_{n-r}}^{+\infty} \ldots \int_{s_{1}}^{+\infty} G_{x}(s) d s d s_{1} \ldots d s_{n-1} \leq \frac{\left(c_{2}-1\right) e R(t)}{4}
$$


Hence,

$$
\begin{aligned}
(U x)(t)+(S y)(t) & \geq \frac{3\left(1-c_{2}\right)}{2 c(t+d)} e R(t)-\frac{x(t+d)}{c(t+d)}+\frac{\left(c_{2}-1\right) e R(t)}{4 c(t+d)} \\
& \geq\left(\frac{3\left(1-c_{2}\right)}{2 c(t+d)}-\frac{1}{c(t+d)}+\frac{c_{2}-1}{4 c(t+d)}\right) e R(t) \\
& \geq e R(t) .
\end{aligned}
$$

Again, in view of (15) and (18), we have

$$
\begin{aligned}
& (U x)(t)+(S y)(t) \\
\leq & \frac{3\left(1-c_{2}\right)}{2 c(t+d)} e R(t)-\frac{x(t+d)}{c(t+d)}-\frac{\left(c_{2}-1\right) e R(t)}{4 c(t+d)} \\
= & \frac{3\left(1-c_{2}\right)}{2 c(t+d)} e R(t)-\frac{2 e}{c(t+d)} \frac{R(t+d)}{R(t)} R(t)-\frac{\left(c_{2}-1\right) e R(t)}{4 c(t+d)} \\
\leq & \left(\frac{3\left(1-c_{2}\right)}{2 c(t+d)}-\frac{7-7 c_{2}-8 c(t+d)}{4 c(t+d)}-\frac{\left(c_{2}-1\right)}{4 c(t+d)}\right) e R(t) \\
\leq & 2 e R(t) .
\end{aligned}
$$

That is, $U x+S y \in X$.

(ii) In view of (12), $U$ is a contraction mapping since it is easy to see that

$$
\frac{1}{R^{2}(t)}|(U x)(t)-(U y)(t)| \leq \frac{1}{c_{1}} \sup _{t \geq \bar{T}} \frac{|x(t)-y(t)|}{R^{2}(t)}
$$

for any $x, y \in X$.

(iii) The operator $S$ is a completely continuous mapping. Indeed, we first note that (13) implies $-c_{2} / c(t) \geq 1$ and (14) implies $-c_{2} / c(t) \leq 8 / 7$. Hence when $t \in[\bar{T}, T)$, $(S x)(t) \geq 3 e R(t) / 2$ and $(S x)(t) \leq(3 / 2)(8 / 7) e R(t) \leq 2 e R(t)$. For $t \in[T,+\infty)$,

$$
\begin{aligned}
(S x)(t) & \geq-\frac{3 c_{2}}{2 c(t+d)} e R(t)+\frac{\left(c_{2}-1\right) e R(t)}{4 c(t+d)} \\
& \geq \frac{e R(t)}{4 c(t+d)}\left(-1-5 c_{2}\right) \geq e R(t),
\end{aligned}
$$

and

$$
\begin{aligned}
(S x)(t) & \leq-\frac{3 c_{2}}{2 c(t+d)} e R(t)-\frac{\left(c_{2}-1\right) e R(t)}{4 c(t+d)} \\
& \leq \frac{\left(-7 c_{2}+1\right) e R(t)}{4 c(t+d)} \leq 2 e R(t) .
\end{aligned}
$$

Therefore the operator $S$ maps $X$ into $X$. The fact that $S$ is continuous and $S X$ is relatively compact can be proved in a manner similar to that in [1] and is omitted. 
By the Krasnoselskii fixed point theorem, there then exists $x \in X$ such that $(U x)(t)+$ $(S x)(t)=x(t)$. Therefore,

$$
x(t)=\frac{3\left(1-c_{2}\right)}{2 c(t+d)} e R(t)-\frac{x(t+d)}{c(t+d)}+\frac{(-1)^{n-r-1}}{c(t+d)} H(t), t \geq T .
$$

It is now clear that $x(t)$ is a nonoscillatory solution of $(1)$ and satisfies

$$
\lim _{t \rightarrow+\infty} \frac{z(t)}{t^{r}}=\frac{3}{2}\left(1-c_{2}\right) e .
$$

By Lemma 2, we have

$$
\lim _{t \rightarrow+\infty} \frac{x(t)}{t^{r}}=\frac{3\left(1-c_{2}\right) e}{2\left(1+c_{0}\right)} \text { and } \lim _{t \rightarrow+\infty} \frac{x(t)}{t^{r-1}}=+\infty .
$$

So $x(t) \in T_{r}(\infty, a)$. In a similar way, we can prove the other case where $K<0$. Our proof is complete.

Theorem 2. Suppose $\lim _{t \rightarrow \infty} c(t)=c_{0}<-1$ and $f$ is superlinear or sublinear. Then $x(t) \in T_{r}(a, 0)$ is a nonoscillatory solution of (1) if, and only if, there is some $K \neq 0$ such that

$$
\int_{t_{0}}^{+\infty} s^{n-r}\left|f\left(s, K g_{1}^{r-1}(s), K g_{2}^{r-1}(s), \ldots, K g_{m}^{r-1}(s)\right)\right| d s<+\infty
$$

The proof is similar to that of Theorem 1, except that the operator $S$ is taken as follows:

$$
(S x)(t)=\left\{\begin{array}{ll}
-\frac{3 c_{2} e R(t)}{2 c(T+d)} & \bar{T} \leq t<T \\
-\frac{3 c_{2} e R(t)}{2 c(t+d)}+\frac{(-1)^{n-r}}{c(t+d)} H(t) & t \geq T
\end{array},\right.
$$

where $H(t)=\int_{T+d}^{t+d} \int_{T}^{s_{n-1}} \ldots \int_{T}^{s_{n-r+2}} \int_{s_{n-r+1}}^{+\infty} \ldots \int_{s_{1}}^{+\infty} G_{x}(s) d s d s_{1} \ldots d s_{n-1}$ and $R(t)=$ $t^{r-1}$ if $r>1$ and $R(t) \equiv 1$ if $r=1$.

\section{References}

[1] Y. H. Chen, Asymptotic behavior of nonoscillatory solutions of higher order neutral delay differential equations, Ann. of Diff. Eqs. 9 (1993), 270-286.

[2] L. W. Wang, Asymptotics of solutions to higher order neutral functional differential equations, Acta Math. Sci. 15 (1995), 153-162 (in Chinese).

Deparment of Computer Science, Guangdong Polytechnic Normal University, Guangzhou, Guangdong 510665, P. R. China.

Department of Mathematics, Tsing Hua University, Hsinchu, Taiwan 30043, R. O. China. 